\title{
La atención y memoria en estudiantes con baja comprensión \\ lectora
}

Maritza Martínez Pérez

merso106@hotmail.com

José Luis Gama Vilchis

Ari7203_2@yahoo.com.mx

Claudia Angélica Sánchez Calderón

Clau_sc2014@hotmail.com

Uriel Ruíz Zamora

Uruizz2276@gmail.com

Universidad Autónoma del Estado de México

Recibido: 28 de abril del 2020

Aceptado: 28 de mayo del 2020

\section{Resumen}

Constantemente se busca dar mejores resultados en los procesos lectores de los estudiantes, en el presente trabajo se aborda el tema desde una postura del proceso psicológico del aprendizaje. La finalidad del estudio es medir el nivel de atención y memoria en estudiantes con bajo desempeño en comprensión lectora. El tipo de estudio es descriptivo. La población de estudio son 93 estudiantes de quinto grado de primarias públicas del valle de Toluca, del Estado de México, entre 9 a 11 años. Se aplicó el test PROLEER para determinar el nivel lector que poseen, una vez identificados a los estudiantes con bajo promedio, se aplicó de forma individual el test de Neuropsi para medir los procesos cognitivos psicológicos, atención y memoria. Para el análisis de los resultados se utiliza estadística descriptiva y diferentes gráficos, para determinar las características de este grupo de niños.

Palabras clave: Comprensión Lectora, atención y memoria. 


\title{
Attention and memory in students with low reading comprehension
}

\begin{abstract}
Constantly seeking to give better results in the student's reading processes, in this work the subject is approached from a position of the psychological process of learning. The purpose of the study is to measure the level of attention and memory in studies with low performance in reading comprehension. The type of study is descriptive. The study population is 93 fifth grade students of public primary schools the Toluca valley, in the State of Mexico, between 9 and 11 years old. The PROLEER test was applied to determine the reading level that they possess, once the students with low average were identified, the Neuropsi test was applied individually to measure the cognitive psychological processes, attention and memory. Descriptive statistics and different graphs are used to analyze the results to determine the characteristics of this group of children.
\end{abstract}

Keywords: Reading Comprehension, attention and memory.

\section{Introducción}

La educación por ser un tema relevante la Organización de las Naciones Unidas para la educación, la Ciencia y la Cultura (UNESCO) y los países miembros de la Organización para la Cooperación y el Desarrollo Económico (OCDE), establecieron objetivos sustentables para el 2030, en educación una de las metas es garantizar una educación de calidad e inclusiva. Un elemento para garantizar la calidad es que las personas tengan conocimientos básicos de alfabetización (lectura y escritura) y reconocer la inclusión como un derecho de todas las personas (UNESCO, 2017).

La situación en México sobre competencia lectora de niños y jóvenes no son alentadores. El resultado del examen de PISA (Programa Internacional de Evaluación de Alumnos) en 2015 sobre habilidad lectora en jóvenes de 15 años está por debajo de la media mundial (INNE, 2016). Así como resultados de la prueba PLANEA (Plan Nacional para la Evaluación del Aprendizaje) del 2015 en niños de $6^{\circ}$ de primaria en el área de Lenguaje y 
Comunicación, se obtuvo que el $49.5 \%$ de los niños se encuentra en el nivel I (logro insuficiente) y un $33.2 \%$ en el nivel II (logro indispensable), se concluye que no se ha podido lograr que todos los estudiantes adquieran las habilidades (INNE, 2015).

\section{Marco teórico}

La competencia lectora es muy amplia incluye tres procesos que son la velocidad, fluidez y comprensión lectora. La velocidad se refiere a cuantas palabras lee la persona por minuto, la fluidez es el ritmo de la lectura en voz alta y la comprensión es entender el texto escrito (SEP 2015). Irrazabal explica que el proceso de comprensión lectora se inicia por entender partes más simples que componen el texto, es decir, palabras y oraciones, de esta forma la persona se forma una representación mental de lo escrito. También hay diferentes niveles de comprensión, en donde la mente utiliza procesos perceptuales y de conceptualización (Irrazabal, 2007).

La relación de comprensión lectora con los procesos cognitivos psicológicos de atención y memoria presentes en el aprendizaje, es retomado por Muelas, quien plantea la necesidad de trabajar los procesos de aprendizaje desde etapas tempranas del desarrollo humano, para que los estudiantes logren ejercitar y tener un mayor potencial en la memoria, que ayuda al aprendizaje y comprensión lectora (Muelas, 2014).

El proceso de aprendizaje a nivel cerebral se explica por medio de procesos cognitivos, resaltando dos funciones principales, la entrada sensorial de la información, y la segunda son las respuestas del organismo a esa información recibida, por medio de acciones de las funciones motoras. Entre estos procesos cognitivos se encuentra la atención, memoria, aprendizaje, percepción, lenguaje y el mecanismo de solución de problemas (Rosselli, et al, 2010).

Rivas describe el proceso de aprender, comienza por mecanismos simples e involuntario del organismo que comienza por captar los estímulos externos, por la percepción de los sentidos, simultáneamente la atención selecciona y focaliza los elementos del medio, según el objetivo que se tenga. La información que se ha recibido, se empieza a almacenar, como primera instancia en la memoria sensorial durante un breve momento, para continuar 
en la memoria a corto plazo, en este tipo de memoria se puede desechar o continuar el proceso, si la experiencia es repetida o se da con mayor precisión, queda en la memoria de largo plazo (Rivas, 2008).

En este proceso resaltaremos dos funciones cognitivas la atención y memoria.

\section{Atención}

Es la capacidad mental, que regula la entrada de estímulos al cerebro, por medio de focalizar nuestros sentidos, seleccionando los elementos que más nos interesan, para que ese estímulo pueda tener la relevancia requerida de nuestro organismo (Estévez, et al., 1997). La atención hace uso de diferentes tareas cognitivas de diferente intensidad, se destaca, darnos cuenta de los elementos del medio, una atención más sostenida cuando comienza una acción y la capacidad de concentración en una tarea específica (Castillo, 2009).

\section{Memoria}

Los autores Etchepareborda y Abad-Mas (2005), definen la memoria y las fases. La memoria es la capacidad de retener información, mediada por el proceso de almacenamiento y evocar información pasada por el proceso de recuperación, que ocurre a nivel neurobiológico. Este proceso complejo es comparado con un archivo, porque en él se guarda la información que percibimos del exterior, por tres pasos fundamentales que son: codificación, almacenamiento y recuperación de la información o evocación.

El primer paso de la codificación, es extraer la información del medio, para darle un código en forma de imágenes, sonidos, sensaciones, ideas, etc., para que estos recuerdos sean guardados. El almacenamiento, ocurre una vez que se ha extraído la información, se va organizando en la mente, por medio de categorías, generando estructuras mentales, que serán almacenadas por un tiempo determinado. La información que ya ha sido almacenada puede ser de tipo visual, auditiva, kinestésica, espacial, emocional y semántica (Téllez, et al, 2009).

La última fase es la de recuperación, se hace uso de la información que ya fue almacenada, según la tarea que lo requiera, será de fácil adquisición si se ha ordenado correctamente. 
En México se han encontrado pocos estudios que vinculen los procesos cognitivos con la mejora de comprensión lectora, Bahena observó la relación entre atención y memoria con los hábitos de sueño, para ver si interfería en el proceso de aprendizaje, se encontró que el ver televisión antes de dormir y dormir pocas horas, eran factores negativos que influían en el desempeño deficiente de la atención y memoria en el proceso de aprendizaje (Bahena, et al, 2019). Un segundo estudio de Esquivel, se aplicó a jóvenes pruebas estandarizadas de amplitud lectora y prueba tipo cloze para medir la relación de la memoria de trabajo con la comprensión lectora, los resultados en estudiantes Universitarios la relación fue alta, en preparatoria la relación fue menor (Esquivel, et al, 2016).

En esta obra se considera fundamental aportar información sobre como los procesos cognitivos influyen en la lectura, por lo que la contribución de los niveles de atención y memoria que poseen los estudiantes con bajo rendimiento en comprensión lectora, ayudará a comprender la importancia de estos procesos para continuar con otras investigaciones y diseño de intervenciones.

\section{Metodología}

El objetivo de la investigación es medir el nivel de atención y memoria en estudiantes con bajo desempeño en comprensión lectora. El método es empírico analítico, con un tipo de estudio descriptivo, para detallar el nivel lector, los procesos cognitivos de atención y memoria, con un diseño transversal, los datos fueron recogidos en dos momentos del ciclo escolar, al inicio y un mes de después. Participaran 93 estudiantes de primarias públicas del Estado de México.

\section{Instrumentos}

Los instrumentos que se aplicaran son dos pruebas:

A) El instrumento empleado fue PROLEER (Matute y González, 2015), tiene como objetivo la detección oportuna de niños con dificultades lectoras. Evalúa los predictores de lectura, compuesta por seis tareas: Pareamiento de Seudopalabras, Pareamiento de No palabras, Sílaba tónica, Pareamiento de Antónimos, Paramiento de Sinónimos y Ortografía. Se aplica de forma grupal, con un tiempo estimado de 15 minutos. 
B) El test Neuropsi, Atención y Memoria (Ostrosky, et al, 2012). Su objetivo es evaluar los procesos cognitivos para poder identificar y dar seguimiento a las dificultades presentes en las áreas de atención y memoria. Su aplicación es de forma individual con un tiempo de 90 minutos. Está dividido en tres áreas que corresponden a la atención, memoria y la última es un total de atención y memoria.

\section{Procedimiento}

La evaluación se hizo en dos momentos diferentes, se inició con la aplicación de PROLEER a los tres grupos de primaria, se evaluó las pruebas para determinar los percentiles alcanzados por los alumnos en lectura, clasificando el desempeño de los alumnos en alto, medio y bajo promedio. De los resultados de PROLEER, se tomó la muestra de 21 alumnos con bajo promedio en comprensión lectora, para aplicar el segundo instrumento de Neuropsi de forma individual, para determinar su nivel de atención y memoria. Se utilizaron medidas de tendencia central, para el tratamiento y análisis de los datos.

\section{Resultados}

Para el análisis de resultados se creó una base de datos de la información recabados de cada una de las dos pruebas y se procesó en SPSS 26.0. Primero se concentró información de los 93 estudiantes de la prueba PROLEER que mide el nivel lector. En la Tabla 1, se presentan los resultados de la prueba PROLEER, clasificando el desempeño de los estudiantes en promedio alto, promedio y promedio bajo.

Tabla 1 . Se muestra el desempeño de la población de estudiantes, 2 tienen promedio alto, 70 obtuvieron un desempeño dentro del promedio y 21 de ellos un promedio bajo.

Nivel Lector de la prueba PROLEER a estudiantes de $5^{\circ}$ de Primaria

\begin{tabular}{lc}
\hline \multicolumn{1}{c}{ Nivel de lectura } & Número de estudiantes \\
& \\
\hline Promedio alto & 2 \\
Promedio & 70 \\
Promedio bajo & 21 \\
\hline
\end{tabular}


En base a los resultados de PROLEER se obtuvo la muestra de 21 estudiantes con promedio bajo en comprensión lectora, a continuación, se valora los resultados que obtuvieron en la prueba de Neuropsi que mide atención y memoria. En la figura 1, se muestran los resultados generales de desempeño de la prueba NEUROPSI, de la suma total de atención y memoria.

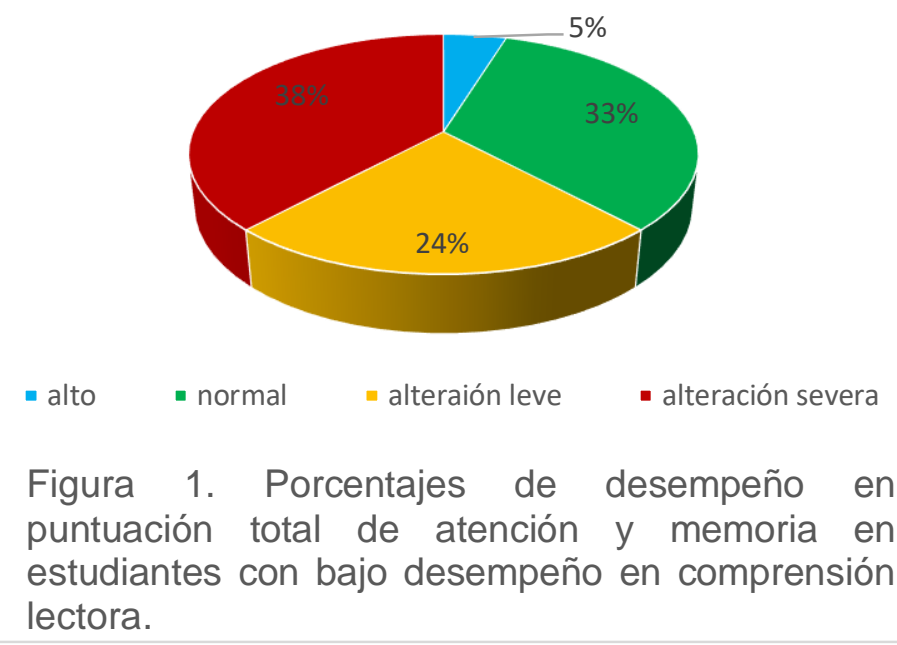

En la figura 1 se aprecia que el 5\% obtuvo desempeño alto, el 33\% se encuentra en normal, el $24 \%$ una alteración leve y el $38 \%$ presentan alteración severa en estas funciones. A continuación, se presentan gráficas de los niveles alcanzados en las dos funciones cognitivas de atención memoria por separado. En la figura 2, se visualizan los porcentajes de desempeño de los estudiantes que obtuvieron en la función cognitiva de atención.

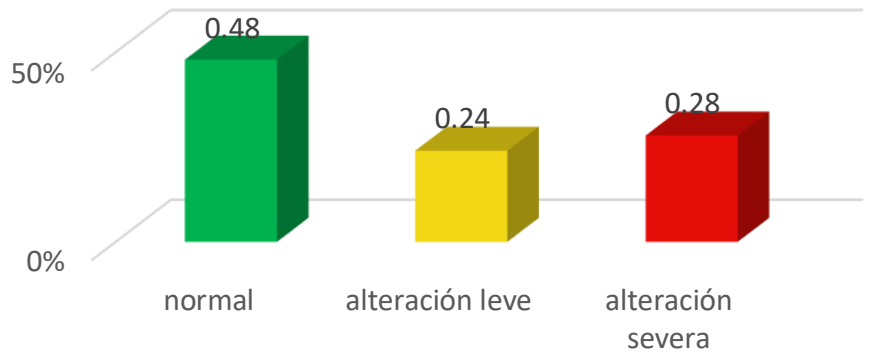

Figura 2. Nivel de atención en estudiantes con bajo desempeño en comprensión lectora

En la Figura 2, se aprecia el desempeño en atención, el 48\% tiene un nivel normal, el 24\% tiene una alteración leve y el 28\% tiene una alteración severa.

Revista RedCA junio-septiembre 2020 ISSN: 2594-2824 Vol. 2 Núm. 7 
La figura 3, se presentan los porcentajes de desempeño de la función cognitiva de memoria de los estudiantes con bajo desempeño en predictores de lectura.

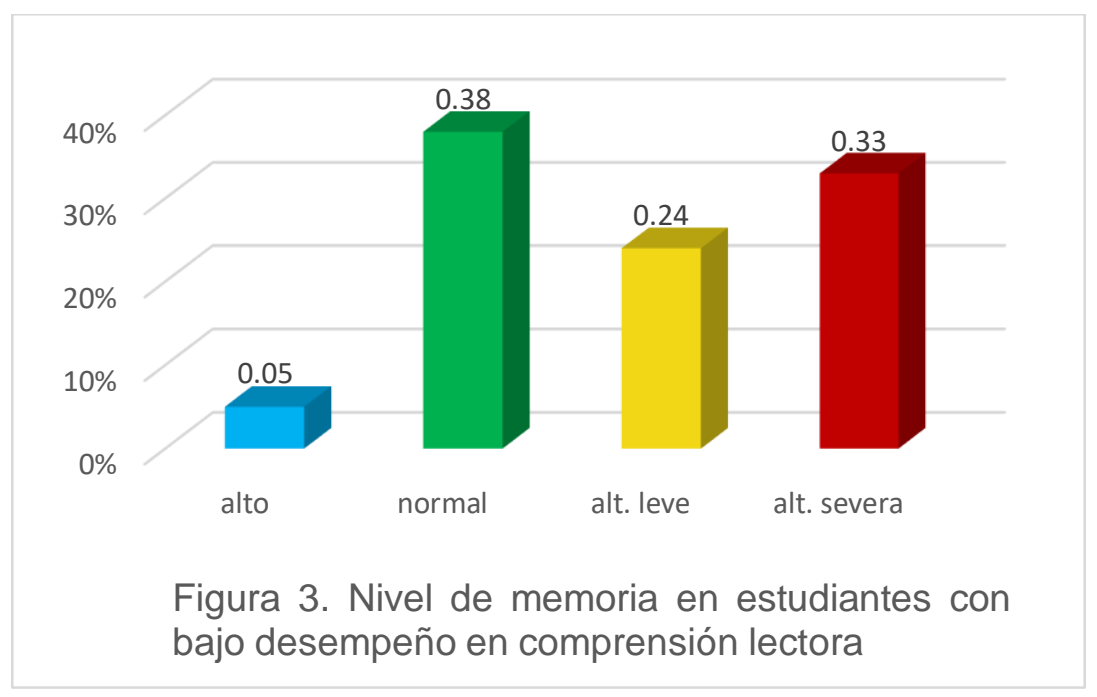

En la figura 3, el 5\% tiene un desempeño alto, el 38\% está en normal, el 24\% tiene una alteración leve y el 33\% posee una alteración severa.

\section{Discusión de resultados.}

El objetivo de esta investigación fue medir el nivel de atención y memoria en estudiantes con bajo desempeño en comprensión lectora. En base a este planteamiento se realiza el análisis de los hallazgos. Se midió el nivel de las funciones cognitivas de atención y memoria en estudiantes con bajo desempeño en comprensión lectora, los resultados indican, que el $62 \%$ presenta alteraciones de leve a severa en las funciones cognitivas de atención y memoria.

Los resultados se pueden comparar con las aportaciones de Fuenmayor y Villasmil, quienes relacionan las funciones cognitivas (percepción, atención y memoria) con la comprensión lectora en Universitarios y concluyen que los estudiantes tienen un deficiente desempeño de las funciones cognitivas, por lo que su comprensión lectora fue pobre (Fuenmayor y Villasmil, 2008).

Los datos encontrados del desempeño de atención en los alumnos con bajo nivel de comprensión lectora, el 52\% presenta una alteración de leve a severa, en aportaciones de Jian y Farquharson que la atención comportamental influye más en la comprensión auditiva y de 
forma indirecta en la comprensión lectora (Jian and Farquharson, 2018). Rangel destaca la importancia de la orientación y el enfoque de atención visual en la lectura (Rangel, et al, 2015). Por lo que se describe la importancia de la atención y memoria en la comprensión lectora.

Los resultados obtenidos en memoria, el 57\% de los estudiantes tiene una alteración de leve a severa, esta función está ligeramente más afectada que la atención, Rodríguez reporta que encontró deficiencias en la memoria operativa, en niños con dificultad de aprendizaje en escritura, igualmente en niños con TDAH (Rodríguez, et al 2009) Y nuevamente Jian and Farquharson mencionan la importancia de la memoria en la comprensión lectora (Jian and Farquharson, 2018).

\section{Conclusiones}

El presente trabajo responde al objetivo planteado, concluyendo la importancia de las funciones psicológica de atención y memoria en la comprensión lectora. En educación básica aún no se le da la importancia suficiente para el desarrollo óptimo de estas funciones para el mejoramiento de la competencia lectora, a lo largo de todo el proceso formativo de niños y jóvenes. Por lo que su atención y desarrollo en diferentes programas son temas de futuras investigaciones. 


\section{Referencias Bibliográficas}

Bahena, G., López, J. y Granados, D. (2019). Hábitos de sueño, memoria y atención en niños escolares. Rev Mex Neuroci, 20 (1), pp 42-49.

Castillo, M. D. (2009). La atención. España: PIRÁMIDE

Esquivel, I., Martínez, W., Córdoba, R. y Reyes, C. (2016). Memoria operativa y lectura comprensiva: medición con pruebas de amplitud lectora y tipo cloze en ámbitos pre universitarios. Apertura, 8 (2): 38-53.

Estévez, A., García, C. y Junqué C. (1997). La atención: una compleja función cerebral. Revista de Neurología, 25 (148).

Etchepareborda, M.C. y Abad-Mas L. (2005) "Memoria de trabajo en los procesos básicos del aprendizaje”. Revista de Neurología, 40 (supl 1) S79-S89.

Fuenmayor, G. y Villasmil, Y. (2008). La percepción, la atención y la memoria como procesos cognitivos utilizados para la comprensión textual. Revista de Artes y Humanidades UNICA, 9 (22): 187-202

Jian, H. and Farquharson, K. (2018). Are working memory and behavioral attention equally important for both reading and listening comprehension? A developmental comparison Language and Reading Research Consortium. Read Writ, 31:1449-1477.

Instituto Nacional para la Evaluación de la Educación (INNE, 2016). México en PISA 2015. 1ra edición. México: INNE.

Instituto Nacional para la Evaluación de la Educación (INNE, 2015). Resultados Nacionales 2015. México: INNE

Irrazabal, N. (2007). Metacomprensión y comprensión lectora. Subjetividad y proceso cognitivos, 10: 43-60. 
Matute, E. y González, A. L. (2014). PROLEER, Escrutinio de predictores de lectura. México: Manual Moderno.

Mulas, A. (2014). La influencia de la memoria y las estrategias de aprendizaje en relación a la comprensión lectora en estudiantes de educación primaria. International Journal of Developmental and Educational Psychology, 6 (1): 343350.

Organización de la Naciones Unidas para la Educación, la Ciencia y la Cultura (UNESCO, 2017). Educación 2030 Declaración de Incheon y Marco de Acción ODS 4. París: UNESCO.

Ostrosky, F., Gómez, M. E., Matute, E., Rosselli, M., Ardila, A. y Pineda, D. (2012). NEUROPSI, Atención y Memoria. México: Manual Moderno $2^{\circ}$ edición.

Rangel, F., García, B. y Galera, C. (2015). Memória de trabalho fonológica, atencao visual e leitura em criancas de $5^{\circ}$ e $6^{\circ}$ séries do ensino fundamental. Estudios de Psicología, 20 (2): 82-91.

Rivas, M. (2008). Procesos cognitivos y aprendizaje significativo. España: Consejería de Educación Comunidad de Madrid.

Rodríguez, C., Álvarez, D., González-Castro, P., García, J., Álvarez, L., Núñez, J., González J. y Bernardo, A. (2009). TDAH y Dificultades de Aprendizaje en escritura: comorbilidad en base a la Atención y Memoria Operativa. Eropean Journal of Education and Psychology, 2 (3):181-198.

Rosselli, M., Matute, E. y Ardila, A. (2010). Neuropsicología del desarrollo infantil. México: Manual Moderno.

Secretaria de Educación Pública (SEP 2015). Toma de lectura, producción de textos escritos y cálculo mental. Herramientas para el Supervisor. México: SEP 
Tellez, A., Téllez, H., Mendoza, M. E., Butcher, E. A, Pacheco, C. C. y Tirado, H. (2002). Atención, aprendizaje y memoria, aspectos psicobiológicos. México:Trillas 